\title{
Evaluación hemodinámica y respuesta al óxido nítrico inhalado ante hipoxia y acidosis en cerdos recién nacidos
}

\author{
MIGUEL MARTELL ${ }^{1}$, FERNANDO BLASINA ${ }^{1,3}$, SALVADOR TELLECHEA ${ }^{1}$, FERNANDO SILVERA ${ }^{1}$, \\ LUCÍA VAAMONDE ${ }^{1,3}$, PABLO BOLIOLI ${ }^{2}$, MARIO MORAES ${ }^{2}$, CAROLINA GODOY ${ }^{1}$ \\ 1. Departamento de Neonatología, Área Básica, Hospital de Clínicas, Montevideo, Uruguay. \\ 2. Departamento de Neonatología Centro Hospitalario Pereira Rossell, Montevideo Uruguay. \\ 3. Departamento de Neuroquímica, Instituto de Investigaciones Clemente Estable.
}

\begin{abstract}
Hemodynamic evaluation and response to inhaled nitric oxyde in newborn piglets

Pulmonary hypertension (HTP) is a frequently pathological situation of maladaptation to the extratuerine life in newborns. Hypoxia and acidosis are key factors capable of evoking the referred situation and can also act as a perpetuator factor of it. Objectives: To evaluate the effect of inhaled nitric oxide (iNO) on pulmonary vascular response to hypoxia and acidosis in newborn pigs. Material and Methods: we performed a series of experimental interventions in newborn pigs, sedated, anesthetized and mechanical ventilation. The experimental protocol was to induce hypoxia in a controlled and progressive $\left(\mathrm{FiO}_{2}\right.$ from 0.21 (baseline) to $0.14,0.10$ or 0.08 , mixing nitrogen with air), between 6 and 10 minutes, evaluating the hemodynamic response. Stabilized response to hypoxia plateau elevation in pulmonary artery pressure (PAP) and systemic administered iNO at $20 \mathrm{ppm}$. By infusion of $\mathrm{HCl} 0.1 \mathrm{~N} \mathrm{i} / \mathrm{v}$ was stable at different $\mathrm{pH}$ values which were reiterated in this series of experiments. Results: we found that the decrease in $\mathrm{FiO}_{2}$ (below 0,21) produces a fall in saturation and an immediate increase in PAP at different $\mathrm{pH}$ and an ERA of iNO for the PAP down near 70\%. However, at lower $\mathrm{pH}$, the presence of acidosis produces a basal level of PAP high relative to $\mathrm{pH} 7.4$, which does not descend iNO. As PH developed at different $\mathrm{FiO}_{2}$ was confirmed that the elevation of the PAP is gradually higher. Conclusion: experimental research in newborn pigs shows that the short-term hypoxic events generate HTP and instability on systemic hemodynamics. Similarly acidosis induces an increase in baseline PAP in this experimental model. Under these conditions the application of iNO decreases the PAP effectively without altering systemic hemodynamics. Its action is more effective at normal $\mathrm{pH}$ than in acidosis.
\end{abstract}

(Key words: Hemodynamics, nitric oxide, anoxia, acidosis, hypertension pulmonary, animals newborn). Arch Pediatr Urug 2009; 80 (4): 262-268

Correspondencia a:

Dr. Miguel Martell

E-mail:migale@multi.com.uy 


\section{RESUMEN}

La hipertensión pulmonar (HTP) es un trastorno de la adaptación a la vida extrauterina de muchos recién nacidos. La hipoxia y la acidosis son factores desencadenantes y perpetuadores de la misma.Objetivos: evaluar el efecto del óxido nítrico inhalado (ONi) a la respuesta vascular pulmonar a la hipoxia y acidosis en cerdos recién nacidos. Material y Método: se diseñó una serie de intervenciones experimentales en cerdos recién nacidos, sedados, anestesiados y en asistencia ventilatoria mecánica. El protocolo experimental consistió en inducir hipoxia en forma controlada y progresiva $\left(\mathrm{FiO}_{2}\right.$ desde 0,21 (basal) hasta 0,$14 ; 0,10$ o 0,08 , mezclando nitrógeno con aire), entre 6 y 10 minutos, valorando la respuesta hemodinámica. Estabilizada la respuesta a la hipoxia con elevación en meseta de la presión arterial pulmonar (PAP) y sistémica, se administró ONi a 20 ppm. Mediante infusión de $\mathrm{HCl} 0,1 \mathrm{~N}$ i/v se estabilizó el pH en diferentes valores en los cuales se reiteraron esta serie de experimentos. Resultados: se encontró que el descenso de la $\mathrm{FiO}_{2}$ (debajo de 0,21) produce una caída de la saturación y un aumento inmediato de la PAP a diferentes $\mathrm{pH}$ y una efectividad del ONi para descender la PAP próximo a 70\%. Sin embargo a pH más bajo la existencia de acidosis genera un nivel basal de PAP elevado con respecto a $\mathrm{pH} 7,4$, que el ONi no desciende. En cuanto a la HTP desarrollada ante diferentes $\mathrm{FiO}_{2}$ se confirmó que la elevación de la PAP es gradualmente más elevada. Conclusión: en la investigación experimental en cerdos recién nacidos se observa que los eventos hipóxicos de breve duración generan HTP e inestabilidad sobre la hemodinamia sistémica. De igual modo la acidosis induce un incremento en la PAP basal en este modelo experimental. En estas condiciones la aplicación de ONi desciende la PAP eficazmente sin alterar la hemodinamia sistémica. Es más efectiva su acción a pH normal que en acidosis.

(Palabras clave: Hemodinámica, óxido nítrico, anoxia, acidosis, hipertensión pulmonar, animales recién nacidos).

Arch Pediatr Urug 2009; 80 (4): 262-268

\section{ESTE TRABAJO LO PUEDE ENCONTRAR EN EXTENSO EN WWW.SciELO.ORG}

